
\title{
is Research Square \\ Could miR34a be as a Potential Biomarker for Doxorubicin Induced Cardiotoxicity?
}

\author{
Eda Caliskan Yildirim ( $\nabla$ caliskan_eda@yahoo.com ) \\ Hacettepe University \\ Emre Gedik \\ Hacettepe University \\ Gurcan Gunaydin \\ Hacettepe University \\ Cem Coteli \\ Hacettepe University \\ Necla Ozer \\ Hacettepe University \\ Aysegül Uner \\ Hacettepe University \\ Deniz Yüce \\ Hacettepe University \\ Alev Turker \\ Hacettepe University
}

\section{Research Article}

Keywords: breast cancer, anthracycline, cardiotoxicity, biomarker, microRNA

Posted Date: October 27th, 2021

DOl: https://doi.org/10.21203/rs.3.rs-962788/v1

License: (c) (1) This work is licensed under a Creative Commons Attribution 4.0 International License.

Read Full License 


\section{Abstract}

No biomarker is currently available for early detection of anthracycline-induced cardiotoxicity. The purpose of this study was to assess whether the plasma levels of microRNA34a (miR34a) could predict cardiotoxicity in breast cancer patients who received anthracycline-based chemotherapy.

Forty-four breast cancer patients who received anthracycline-based chemotherapy for the first time were included in the study. Before and after taking chemotherapy, patients were examined for cardiac troponinI, miR34a, and precursor miR34a levels, and echocardiographic strain analyses were performed.

There was a statistically significant increase in troponin-I, miR34a, and pre-miR34a levels after treatment with anthracyclines. The mean increase in miR34a and pre-miR34a was 2.5 and 2.3 fold, respectively. Echocardiographic analysis of patients showed a significant decrease in global longitudinal strain (GLS) measurements compared to the baseline after anthracycline treatment. An increase in the levels of miR34a/pre-miR34a was detected in patients who were estimated to have cardiac damage according to GLS change, but this increase was not statistically significant.

After doxorubicin treatment, an increase in miR34a level in plasma was demonstrated without correlation with cTn-I and GLS. A higher miR34a/pre-miR34a ratio was detected in patients with myocardial deformation than in those without myocardial deformation, but it was not statistically significant.

\section{Introduction}

Breast cancer globally accounts for $24.2 \%$ of all cancers in women ${ }^{1}$. In recent years, advances in breast cancer treatment modalities have significantly improved patient survival ${ }^{2}$. Anthracyclines are still the mainstay of breast cancer treatment. Doxorubicin is one of the most widely used chemotherapeutics of the anthracycline family ${ }^{3}$. The most critical side effect of doxorubicin is cardiotoxicity, which can be seen in a spectrum ranging from subclinical ventricular dysfunction to severe heart failure, which may result in mortality. Therefore, the early recognition of cardiotoxicity is essential to prevent ventricular complications ${ }^{4}$.

Cardiac imaging, in particular echocardiography, plays a central role in the assessment of cardiotoxicity, which is defined in many guidelines based on the reduction of the left ventricular ejection fraction $(\text { LVEF })^{5,6}$. However, normal LVEF does not rule out myocardial dysfunction ${ }^{6}$. Also, a significant reduction in LVEF is noticed after a large amount of myocardial damage has occurred ${ }^{7}$. Therefore, assessing cardiotoxicity with LVEF is not sufficient for early diagnosis and timely intervention to prevent cardiac dysfunction.

Global longitudinal strain (GLS) has arisen as a new myocardial deformation parameter measured by speckle tracking echocardiography to identify subclinical ventricular dysfunction ${ }^{8}$. Several studies have demonstrated that a decrease in GLS can accurately indicate potential cardiotoxicity ${ }^{9,10}$. Recently, many 
guidelines have recommended the assessment of GLS to detect anthracycline-associated subclinical left ventricular dysfunction ${ }^{5,11}$.

Cardiac troponins (cTn-I/cTn-T) are widely used circulating markers to identify early myocardial injury due to anthracyclines ${ }^{12}$. Although there is no consensus between cardiology and oncology societies on the use of cardiac troponin as a biomarker, the position statement from the European Society of Cardiology (ESC) considers cTn based on patients' cardiovascular risk status ${ }^{13}$. There is an unmet need for biomarkers to detect cardiotoxicity at an early stage when it is reversible. In recent years, several studies have investigated the role of microRNAs in anthracycline-induced cardiotoxicity ${ }^{14}$.

MicroRNAs, known as small noncoding RNAs that consist of 22-26 nucleotides, play a crucial role in regulating the expression of many genes ${ }^{15}$. MicroRNA34a (miR34a) modulates several cellular processes, such as apoptosis, differentiation, senescence, and energy metabolism ${ }^{16,17}$, and plays a regulator role in cardiac injury and its repair ${ }^{18-20}$. The increase in the tissue and plasma levels of miR34a following doxorubicin exposure in animal models has strengthened the belief that it may be both a biomarker and a therapeutic target ${ }^{21-24}$.

Recent studies indicate that different molecules play a role in miRNA biogenesis ${ }^{25,26}$. The difference between pre-miRNA and miRNA levels refers to the posttranscriptional modification of miRNA biogenesis. This difference may be due to various genetic mutations or signal pathway defects ${ }^{27-29}$. In this context, it is important to compare pre-miRNA and mature miRNA levels and determine the difference between the two transcripts to understand the functional effect of the target miRNA.

This is the first human study in which the plasma miR34a level was evaluated together with precursor pre-miR34a and GLS as early cardiotoxicity markers. We aimed to reveal the relationship between early myocardial deformation and plasma cTn-I, pre-miR34a, and miR34a.

\section{Results}

\section{Demographic characteristics of breast cancer patients}

The demographic characteristics of the 44 patients who completed the study are given in Table 1. All patients had doxorubicin as an anthracycline in the CT protocol. The cumulative doxorubicin dose (mean $+\mathrm{SD}$ ) was calculated as $242 \pm 26 \mathrm{mg} / \mathrm{m}^{2}$. Eighty-one percent of the patients received the AC regimen, and $17 \%$ received the CAF regimen. Only one patient received the TAC regimen. 
Table 1

Characteristics of patients.

\begin{tabular}{|c|c|}
\hline Characteristics & Patients $(n=44(\%))$ \\
\hline Age $($ mean $\pm S D)$ & $52 \pm 10$ \\
\hline $\mathrm{BMI}($ mean $\pm \mathrm{SD})$ & $26.5 \pm 4.1$ \\
\hline Smokers [n (\%)] & $9(22)$ \\
\hline \multicolumn{2}{|l|}{ Menopause status [n (\%)] } \\
\hline Premenopause & $19(43)$ \\
\hline Postmenopause & $25(57)$ \\
\hline \multicolumn{2}{|l|}{ Comorbidities [n (\%)] } \\
\hline Diabetes & $3(7)$ \\
\hline Hypertension & $9(21)$ \\
\hline Vascular disease & $0(0)$ \\
\hline Doxorubicin cumulative dose- $\mathrm{mg} / \mathrm{m}^{2}($ mean $\pm \mathrm{SD})$ & $242.3 \pm 26.72$ \\
\hline \multicolumn{2}{|l|}{ Doxorubicin dose [n (\%)] } \\
\hline$<200 \mathrm{mg} / \mathrm{m}^{2}$ & $4(10)$ \\
\hline $200-400 \mathrm{mg} / \mathrm{m}^{2}$ & $40(90)$ \\
\hline \multicolumn{2}{|l|}{ Cardiovascular risk status [n (\%)] } \\
\hline Low & $20(45)$ \\
\hline Moderate & $19(44)$ \\
\hline High & $5(11)$ \\
\hline LVEF $\leq 55 \%$ and decrease of LVEF $\geq 10 \%[n(\%)]$ & $2(5)$ \\
\hline
\end{tabular}

\section{Baseline clinical, pathological, and treatment features of the breast cancer patients}

The pathologic characteristics of breast cancer patients and their stages are shown in Table 2. While 63\% of the patients received adjuvant treatment, $20 \%$ received neoadjuvant chemotherapy for local advanced disease, and $17 \%$ received treatment for metastatic disease. 
Table 2

Tumor Characteristics.

\begin{tabular}{|c|c|}
\hline Characteristics & Primary breast cancer patients $(n=44)$ \\
\hline \multicolumn{2}{|c|}{ Histological subgroups [n (\%)] } \\
\hline IDC & $32(73)$ \\
\hline ILC & $1(2)$ \\
\hline Mixed (IDC +ILC) & $9(20)$ \\
\hline Other & $2(5)$ \\
\hline \multicolumn{2}{|l|}{ Tumor grade [n (\%)] } \\
\hline 2 & $17(39)$ \\
\hline 3 & $24(61)$ \\
\hline \multicolumn{2}{|c|}{ Molecular subtype [n (\%)] } \\
\hline Luminal A & $9(20)$ \\
\hline Luminal B & $22(50)$ \\
\hline HER2+ & $7(16)$ \\
\hline Basal & $6(14)$ \\
\hline Ki67\% ( mean \pm SD) & $34 \pm 25$ \\
\hline \multicolumn{2}{|l|}{ T diameter [n (\%)] } \\
\hline$<2 \mathrm{~cm}$ & $4(15)$ \\
\hline $2-5 \mathrm{~cm}$ & $21(73)$ \\
\hline$>5 \mathrm{~cm}$ & $4(12)$ \\
\hline \multicolumn{2}{|c|}{ Lymph node involvement [n (\%)] } \\
\hline No & $9(30)$ \\
\hline N1 & $10(33)$ \\
\hline N2 & $8(27)$ \\
\hline N3 & $3(10)$ \\
\hline \multicolumn{2}{|l|}{ Metastasis [n (\%)] } \\
\hline MO & $36(82)$ \\
\hline M1-bone & $5(11)$ \\
\hline M1-visceral organ & $3(7)$ \\
\hline
\end{tabular}




\begin{tabular}{|ll|}
\hline Characteristics & Primary breast cancer patients $(\mathbf{n}=\mathbf{4 4})$ \\
\hline Lymphovascular invasion presence $[\mathrm{n}(\%)]$ & $8(29)$ \\
Stage $[\mathrm{n}(\%)]$ & \\
1 & 0 \\
2 & $18(41)$ \\
3 & $18(41)$ \\
4 & $8(18)$
\end{tabular}

\section{Echocardiographic parameters that changed after treatment}

All patients included in the study were evaluated echocardiographically before and after treatment, and tissue Doppler imaging and strain analyses were performed. Left ventricular global longitudinal strain (GLS) indicating myocardial deformity, tricuspid annular planar systolic excursion (TAPSE) showing right ventricular function, and mitral peak early diastolic velocities $(\mathrm{E})$ assessing diastolic functions were obtained from the peak early myocardial diastolic velocity (é) and septal E/é parameters, are shown in Table 3. Additionally, the change in cardiac troponin-l levels with treatment is also included in the table.

Table 3

Cardiac parameters that changed with treatment.

\begin{tabular}{|llll|}
\hline & Visit 1 (V1) & Visit 2 (V2) & P value \\
\hline Troponin-I (ng/ml) & $0.007 \pm 0.011$ & $0.061 \pm 0.049$ & $<\mathbf{0 . 0 0 1}$ \\
\hline LVGLS (\%) & $19.84 \pm 3.15$ & $17.92 \pm 3.72$ & $\mathbf{0 . 0 0 1}$ \\
\hline RVGLS (\%) & $18.22 \pm 2.3$ & $17.90 \pm 2.8$ & 0.472 \\
\hline TAPSE $(\mathrm{mm})$ & $24 \pm 3$ & $22 \pm 3$ & $\mathbf{0 . 0 0 2}$ \\
\hline Septal E/e & $6 \pm 3$ & $7 \pm 3$ & $\mathbf{0 . 0 0 5}$ \\
\hline Lateral E/e & $5 \pm 2$ & $6 \pm 3$ & $\mathbf{0 . 0 1 5}$ \\
\hline LVEF $(\%)$ & $64 \pm 0.5$ & $62 \pm 0.5$ & $<\mathbf{0 . 0 0 1}$ \\
\hline
\end{tabular}

( LVGLS: left ventricular global longitudinal strain, RVGLS: right ventricular global longitudinal strain, TAPSE: tricuspid annular planar systolic excursion ,LVEF:left ventricular ejection fraction)

\section{mir34a and pre-miR34a expression changes after treatment}

Expression of miR34a before and after treatment was calculated as a fold change according to the Livak model, as previously described in the materials and methods section. As shown in Figure 2, treatment resulted in a statistically significant increase in both miR34a and pre-miR34a expressions. When the miR34a/pre-miR34a ratio was examined, it was observed that the miR34a level was significantly higher than the level of its precursor. 


\section{Comparison of the myocardial deformity of the groups of patients}

As recommended in the 2020 position statement from the $\mathrm{ESC}^{6}$, we examined patients in two groups: those with a GLS change over $15 \%$ and those with a change below the $15 \%$ threshold. Changes in the echocardiographic parameters, cTn-I levels, and plasma miR34a expressions of the two groups are compared in Table 4. LVGLS analysis of a patient is demonstrated in Figure 3.

Table 4

Comparison of patients with and without myocardial deformation

\begin{tabular}{|llll|}
\hline & $\begin{array}{l}\Delta G L S<15 \\
\mathbf{N}=\mathbf{2 8}\end{array}$ & $\begin{array}{l}\Delta \mathrm{GLS}>15 \\
\mathbf{N}=\mathbf{1 3}\end{array}$ & P value \\
\hline $\begin{array}{l}\text { Cumulative doxorubicin dose } \\
\text { (mean } \pm \text { SD) }\end{array}$ & $241 \pm 29$ & $244 \pm 20$ & 0.732 \\
\hline cTn-I-V2 (median (IQR)) & $0.04(0.039)$ & $0.062(0.057)$ & $\mathbf{0 . 0 2 5}$ \\
\hline TAPSE (mean \pm SD) & $1.14 \pm 3.41$ & $3.31 \pm 2.90$ & 0.055 \\
\hline Septal E/e (mean \pm SD) & $1.07 \pm 1.76$ & $0.69 \pm 2.98$ & 0.612 \\
\hline Lateral E/e (mean \pm SD) & $0.79 \pm 1.95$ & $1.0 \pm 2.31$ & 0.759 \\
\hline LVGLS-V2 (mean \pm SD) & $19.27 \pm 3.46$ & $15.43 \pm 2.88$ & 0.002 \\
\hline miR34a (median (IQR)) & $1.82(3.55)$ & $1.64(0.82)$ & 0.889 \\
\hline Pre-miR34a (median (IQR)) & $1.18(1.81)$ & $1.13(1.82)$ & 0.427 \\
\hline miR34a/pre-miR34a (median (IQR)) & $1.61(1.49)$ & $2.58(5.23)$ & 0.287 \\
\hline $\begin{array}{l}\text { (cTn-l:cardiac troponin-I, TAPSE: tricuspid annular planar systolic excursion, LVGLS: left ventricular } \\
\text { global longitudinal strain) }\end{array}$ & & \\
\hline
\end{tabular}

\section{Relationship between doxorubicin dose, troponin - I, miR34a and echocardiographic parameters}

In the evaluation of cardiac deformity according to the GLS change, there was no significant difference in the cumulative doxorubicin dose between those with deformity and those without. However, there was a moderate correlation between cumulative doxorubicin doses and TAPSE $\left(r^{2}=545 p<0.001\right)$ in Figure 4 . As the cumulative dose of doxorubicin increases, the change in TAPSE increases. A weak-moderate correlation was demonstrated between TAPSE-V2 and RVGLS-V2. $\left(r^{2}=354 p=0.023\right)$. There was also a 
moderate negative correlation between LVGLS-V2 and change in TAPSE $\left(r^{2}=395 p=0,016\right)$. Any correlation between cTn-I, miR34a and GLS change could not be found.

\section{Discussion}

To our knowledge, this is the first study to evaluate miR34a, its precursor pre-miR34a, cTn-I, and GLS as early cardiotoxicity markers in breast cancer patients receiving doxorubicin. Our results indicate that plasma miR34a, pre-miR34a, and cTn-I levels for the entire group increased significantly after doxorubicin administration compared to the baseline measurements.

It has been shown that the miR34 family members and miR34a are upregulated in cardiomyocytes when they are exposed to stress, such as myocardial infarction, and contribute to age-dependent cardiac damage ${ }^{18}$. Desai et al. assessed miR34a expressions in doxorubicin-exposed mouse myocardial tissue and showed that miR34a is upregulated in the heart at the very early stages before cardiomyocyte necrosis, unlike troponin ${ }^{23}$. Piegari et al. have reported an increase in miR34a expression, not only in cardiac cells and cardiac progenitor cells (CPC), but also in rat plasma after doxorubicin administration ${ }^{21}$. The demonstration of increased plasma miR34a expression in this study has raised the question of whether miR34a could be a biomarker in doxorubicin-related cardiotoxicity. The first human study exploring whether miR34a could be a cardiotoxicity marker was undertaken by Fréres and his colleagues. This study was designed to reveal circulating microRNA changes in patients with locally advanced breast cancer after neoadjuvant therapy. However, as a secondary outcome, a correlation was found between the change in CTn-T level and miR34a expression after epirubicin treatment ${ }^{30}$. A significant positive correlation was demonstrated at 6 months after doxorubicin exposure in a small group of triple negative breast cancer ${ }^{31}$.

While our study was in progress, Fréres et al. was investigating circulating cardiac biomarkers in patients treated with epirubicin for locally advanced breast cancer. In their study, epirubicin was used as an anthracycline, and the levels of miR34a were evaluated four times: before treatment, after 2 cycles of anthracycline-based chemotherapy, at the end of the chemotherapy and three months after completion of treatment with epirubicin. There was a significant increase in the levels of miR34a in all measurements after epirubicin exposure, and the maximum level occurred after 2 cycles of anthracycline-based chemotherapy. However, no correlation was found between the miR34a levels, LVEF, and cardiac troponin $\mathrm{T}^{32}$. In two studies conducted by Fréres and colleagues, the mean fold change in miR34a expression was higher than in our study. The difference in the mean fold change of miR34a between the studies may be because samples were taken at different times. Another reason may be that $63 \%$ of our patient population was composed of patients receiving doxorubicin as an adjuvant treatment after surgery. In a group of patients who received doxorubicin for neoadjuvant treatment, plasma miR34a expression and the miR34a/ pre-miR34a ratio were higher than in other patients, but this was not statistically significant. Pre-miR34a expression was significantly lower in patients with metastatic disease compared to other 
patients. In a preclinical study, miR34a expression was found to be much lower in metastatic breast cancer cells than non-metastatic cells ${ }^{33}$.

Mir34a is a well-known tumor suppressor miRNA that is transcriptionally regulated by $\mathrm{p} 53$ in response to DNA damage ${ }^{34,35}$. DNA damage caused by anthracyclines and $\mathrm{p} 53$ activation may explain miR-34a upregulation following anthracycline treatment. There are also different activation mechanisms for miR34a. Salzman et al. have demonstrated that there is a pool of existing transcribed miR34a, waiting for rapid activation by phosphorylation as a rapid response to DNA damage ${ }^{36}$. This form of activation of miR34a can explain why we found higher plasma miR34a levels than its precursor.

According to the position statement from the ESC position ${ }^{5}$, patients with possible myocardial deformation (an LVGLS change $>15 \%$ ) and patients without deformation (an LVGLS change $<15 \%$ ) were compared in terms of miR34a, pre-miR34a, cTn-I, septal and lateral e / é,TAPSE, and LVEF. Patients with potential myocardial deformation had significantly higher cTn-I levels than the others. The change in TAPSE was also remarkable, but not statistically significant. The miR34a/pre-miR34a ratio was higher in patients with an LVGLS change greater than $15 \%$. Although these findings support our hypotheses, some of our results did not achieve statistical significance. This suggests that the power of the study is insufficient and deserves consideration in larger populations.

In this study, the correlation between cumulative anthracycline doses and myocardial damage parameters was also examined. Our entire study population received a modest dose of doxorubicin in the range of $200-300 \mathrm{mg} / \mathrm{m}^{2}$. There was no difference between patients with and without myocardial deformation in terms of cumulative doxorubicin dose. We demonstrated a positive correlation between cumulative doxorubicin dose and change in TAPSE. It has been shown in two previous studies that TAPSE decreased after doxorubicin treatment ${ }^{37,38}$. There was a significant decrease in TAPSE in our study, but its level was still in the normal range. While effects of doxorubicin on the left ventricle are established, data on its effects on the right ventricle remain scarce ${ }^{39}$. There is a growing body of data on the longitudinal strain analysis of $\mathrm{RV}^{40}$. A study assessing lymphoma patients treated with doxorubicin reported that RVGLS decreased six months after doxorubicin administration ${ }^{41}$. We assessed RVGLS three months after treatment and could not find a significant difference between the $\mathrm{V} 1$ and $\mathrm{V} 2$ values for RVGLS. However, a correlation was shown between TAPSE-V2 and RVGLS-V2. In line with these arguments, the evaluation of right ventricular function should not be neglected in patients receiving doxorubicin.

The small number of patients and the short follow-up period are the most critical limitations of this innovative study. None of the patients included in the study developed heart failure symptoms, and only two patients had asymptomatic decline of LVEF. Long-term follow-up of these patients is necessary to determine whether heart failure is associated with doxorubicin. Another limitation is that the sample collection time in which miR34a and cTn-I were studied varied within the study. 
We examined miR34a, pre- miR34a, and the miR34a/miR34a ratio as early biomarkers of doxorubicininduced cardiotoxicity in a heterogeneous breast cancer population that included different molecular subtypes and patients at different stages. After doxorubicin treatment, an increase in miR34a level in plasma was demonstrated without correlation with cTn-I and GLS. A higher miR34a/pre-miR34a ratio was detected in patients with myocardial deformation than in those without myocardial deformation, but it was not statistically significant. Long-term studies are needed in different cancer patient groups receiving anthracycline to understand the role of these parameters as early cardiotoxicity biomarkers.

\section{Materials And Methods}

\section{Population}

This study's protocol, which was designed as a prospective cohort study, was approved by local clinical research ethics committee. Fifty-one breast cancer patients who applied to the our oncology department between May 2017 and December 2017 to receive anthracycline-based chemotherapy for the first time were included. All patients gave their consent to participate in the study. Patients with advanced heart failure (New York Heart Association (NYHA) Stage II and further heart failure), a history of myocardial infarction, and renal failure requiring renal replacement were excluded from the study.

The patient flow chart is presented in Figure 1. A total of 51 patients were assessed according to the study criteria. Two of these patients were excluded because of poor echogenicity. After the first visit, two patients withdrew their consent, one patient died, one patient's chemotherapy protocol was changed by her oncologist, and one changed her hospital.

All patients included in the study received either a doxorubicin-cyclophosphamide (AC), cyclophosphamide-doxorubicin-fluorouracil (CAF), or paclitaxel-doxorubicin-cyclophosphamide (TAC) CT protocol. The patients' physicians determined the CT protocols, and protocol selection was not intervened. All patients were evaluated twice: before the first doxorubicin administration and 3-6 weeks after the last doxorubicin administration. During these evaluations, symptoms related to cardiac dysfunction, echocardiography with strain analysis, serum troponin-I levels, plasma miR34a, and premiR34a (a precursor of miR34a) measurements were assessed. The second evaluation was performed in such a manner as to avoid interrupting the treatment plan prior to radiotherapy or CT treatments.

\section{Analysis of echocardiography data}

A comprehensive two-dimensional echocardiography examination was performed in the left lateral position using an M4S-RS probe (Vivid S6, GE Medical Systems, Horton, Norway) at baseline and after the completion of doxorubicin treatment. Echocardiography images were obtained from the parasternalshort axis, the parasternal-long axis, as well as the apical2- and 4-chamber views. Echocardiographic measurements were made based on the criteria recommended by the European Association of Cardiovascular Imaging (EACVI) ${ }^{42}$. 
Cardiotoxicity was defined as LVEF reduced to below $50 \%$ or a greater than $10 \%$ reduction from baseline to the lower limit of normal, which is recommended as $54 \%$ for women and $52 \%$ for men by ESC and $\mathrm{EACVI}{ }^{5}$.

LVEF was measured using the biplane Simpson's method. Tricuspid annular plane systolic excursion (TAPSE) was used to assess right ventricular (RV) function. The mitral peak velocity of early filling(E)and the early diastolic velocity of the lateral and septal mitral annulus $\left(E^{\prime}\right)$ was calculated. The $E / E$ ' ratio was also recorded as a reliable index of left ventricular filling pressure. Each measurement was obtained after at least three consecutive cardiac cycles.

\section{Measurement of myocardial strain}

The standard three apical views were used to obtain global longitudinal peak left ventricular strain (LV GLS), and apical four chamber views were used for global longitudinal peak right ventricular strain (RV GLS). A strain analysis was performed using the Echopac PC version BT13 software. According to the position paper from the ESC, a decrease in LV GLS of greater than $15 \%$ was considered predictive of cardiotoxicity ${ }^{5}$.

\section{Measurement of troponin-I levels}

The serum troponin-l level was measured in two hours at our hospital's biochemistry laboratory after blood samples were taken. This study was performed by an immunoassay method using a monoclonal antibody specifically designed for cardiac Tnl.

\section{Study of miR-34a and pre-miR-34a levels and RNA isolation}

A total of 88 samples were collected from 44 patients in visit 1 and visit 2, and RNA isolation was performed using the miRNeasy Serum/Plasma Kit (Cat. No 217184, QIAGEN, gMBH, D-40724 Hilden, Germany) in accordance with the manufacturer's instructions. The quantities of RNA obtained were measured using 260/230 (an inorganic contaminant) and 260/280 (an organic solvent) values from a $1.5 \mu \mathrm{l} \mathrm{sample} \mathrm{in} \mathrm{a} \mathrm{nano-drop} \mathrm{device.} \mathrm{A} \mathrm{sample} \mathrm{of} \mathrm{three} \mathrm{patients} \mathrm{with} \mathrm{nano-drop} \mathrm{measurement} \mathrm{RNA} \mathrm{levels}$ below $10 \mathrm{ng} / \mu \mathrm{l}$ was excluded from the study. We proceeded to the complementary DNA (cDNA) synthesis phase with 82 samples from 41 patients with no DNA contamination by measurement. cDNA synthesis was performed according to the protocol for the miScript II RT Kit (Cat No. ID: 218161, QIAGEN, gMBh, D40724, Hilden, Germany). cDNA reverse transcriptase PCR results were obtained for $20 \mathrm{ng} / \mu \mathrm{l}$ cDNA. The cDNA samples were prepared according to the protocol for the miScript SYBR Green PCR Kit (Cat No. ID: 218075, QIAGEN). Two target gene primers (miR34a-5p and pre-miR34a) and one reference gene primer (RNU6) were used for two samples from each patient. qPCR results were obtained using the Rotor-Gene 1.7.94 program. The value of $r^{2}$ was greater than 99 , and the activity was in the range of $90-110 \%$ for all test results (14 runs in total). All samples were triplicated, and the expression of the targeted miR34a-5p and pre-miR34a were analyzed. The $\mathrm{Ct}$ (cycle threshold) values determined based on the threshold value were analyzed. The changes in the targeted miR34a and pre-miR34a expressions after anthracycline treatment in the direction of the data obtained by the real-time PCR method were compared. This 
comparison was determined by normalizing the targeted miRNAs with the expression of the reference gene (RNU6). Real time PCR data were calculated using the Livak model $\left(2^{-\Delta \Delta C t}\right)^{43}$

\section{Statistical analysis}

In this analysis, the mean and standard deviation were the descriptive statistics of choice for variables with a normal distribution and for continuous variables, and the median and interval were determined for non-normal distributions or ordinal variables. Categorical variables were described using numbers and percentages. A student's t-test was used for independent groups if the continuous variables were normally distributed in the binary comparisons, and a Mann-Whitney $U$ test was used if the data were not normally distributed. A chi-square test was used for categorical variables. A Spearman correlation test was used to investigate the correlations between the echocardiographic parameters and the troponin-I and miR34a levels. A Wilcoxon test was used for non-normal distribution dependent variables.

The program SPSS for Windows (version 20 package) was used for statistical analysis, and a $p \leq 0.05$ was considered statistically significant.

\section{Declarations}

Author for correspondence: Eda Caliskan Yildirim (ORCID ID: 0000-0003-0785-0238)

\section{Contact Information:}

Dokuz Eylul Medicine Faculty, Department of Medical Oncology, Mithatpasa Street No:1606, Balcova/IZMIR

caliskan_eda@yahoo.com

Acknowledgements: None.

Competing Interest: The authors declare no competing interests.

Data Availability: Data available on request due to privacy/ethical restrictions

Financial Disclosure: Supported by Hacettepe University Scientific Research Committee(SRC Project No:THD-2017-14885)

Information pertaining to writing assistance: None.

Ethical disclosure: The authors state that they have obtained appropriate institutional review board approval or have followed the principles outlined in the Declaration of Helsinki for all human or animal experimental investigations. The study was approved byHacettepe University Non-Interventional Clinical Research Ethics Committee (decision number: G0 17/319-18)

Concent to Participate: Informed consent has been obtained from the participants involved. 


\section{Author Contributions:}

1. Eda Caliskan Yildirim: Primary author, design of the study, data collection, data analysis, writing of manuscript.

2. Emre Gedik: performed experiments, data collection, data analysis.

3. Gurcan Gunaydin: data interpretation.

4. Cem Coteli: data collection.

5. Necla Ozer: data interpretation.

6. Aysegul Uner: data interpretation

7. Deniz Yuce:data analysis

8. Alev Turker: data interpretation

\section{References}

1. Ferlay, J. et al. Estimating the global cancer incidence and mortality in 2018: GLOBOCAN sources and methods. Int J Cancer, 144, 1941-1953 https://doi.org/10.1002/ijc.31937 (2019).

2. Curigliano, G. et al. Cardiotoxicity of anticancer treatments: epidemiology, detection, and management. CA: a cancer journal for clinicians, 66, 309-325 (2016).

3. Raj, S., Franco, V. I. \& Lipshultz, S. E. Anthracycline-induced cardiotoxicity: a review of pathophysiology, diagnosis, and treatment. Current treatment options in cardiovascular medicine, 16, 1-14 (2014).

4. Lotrionte, M. et al. Review and meta-analysis of incidence and clinical predictors of anthracycline cardiotoxicity. The American journal of cardiology, 112, 1980-1984 (2013).

5. Čelutkienè, J. et al. Role of cardiovascular imaging in cancer patients receiving cardiotoxic therapies: a position statement on behalf of the $\mathrm{H}$ eart $\mathrm{F}$ ailure $\mathrm{A}$ ssociation (HFA), the $\mathrm{E}$ uropean $\mathrm{A}$ ssociation of $\mathrm{C}$ ardiovascular I maging (EACVI) and the Cardio-Oncology $\mathrm{C}$ ouncil of the $\mathrm{E}$ uropean S ociety of C ardiology (ESC). European journal of heart failure, 22, 1504-1524 (2020).

6. Čelutkienè, J. et al. Innovative imaging methods in heart failure: a shifting paradigm in cardiac assessment. Position statement on behalf of the Heart Failure Association of the European Society of Cardiology. European journal of heart failure, 20, 1615-1633 (2018).

7. Cardinale, D. et al. Anthracycline-induced cardiomyopathy: clinical relevance and response to pharmacologic therapy. Journal of the American College of Cardiology, 55, 213-220 (2010).

8. Thavendiranathan, P. et al. Use of myocardial strain imaging by echocardiography for the early detection of cardiotoxicity in patients during and after cancer chemotherapy: a systematic review. Journal of the American College of Cardiology, 63, 2751-2768 (2014).

9. Negishi, K. et al. Use of speckle strain to assess left ventricular responses to cardiotoxic chemotherapy and cardioprotection. European Heart Journal-Cardiovascular Imaging, 15, 324-331 (2014). 
10. Charbonnel, C. et al. Assessment of global longitudinal strain at low-dose anthracycline-based chemotherapy, for the prediction of subsequent cardiotoxicity. European Heart JournalCardiovascular Imaging, 18, 392-401 (2017).

11. Plana, J. C. et al. Expert consensus for multimodality imaging evaluation of adult patients during and after cancer therapy: a report from the American Society of Echocardiography and the European Association of Cardiovascular Imaging. European Heart Journal-Cardiovascular Imaging, 15, 10631093 (2014).

12. Cardinale, D., Biasillo, G., Salvatici, M., Sandri, M. T. \& Cipolla, C. M. Using biomarkers to predict and to prevent cardiotoxicity of cancer therapy. Expert Review of Molecular Diagnostics, 17, 245-256 https://doi.org/10.1080/14737159.2017.1283219 (2017).

13. Pudil, R. et al. Role of serum biomarkers in cancer patients receiving cardiotoxic cancer therapies: a position statement from the Cardio-Oncology Study Group of the Heart Failure Association and the Cardio-Oncology Council of the European Society of Cardiology. European Journal of Heart Failure, 22, 1966-1983 (2020).

14. Ruggeri, C., Gioffré, S., Achilli, F., Colombo, G. I. \& D’Alessandra, Y. Role of microRNAs in doxorubicininduced cardiotoxicity: an overview of preclinical models and cancer patients. Heart failure reviews, 23, 109-122 (2018).

15. Creemers, E. E., Tijsen, A. J. \& Pinto, Y. M. Circulating microRNAs. Circulation research, 110, 483-495 (2012).

16. Chen, F. \& Hu, S. J. Effect of microRNA-34a in cell cycle, differentiation, and apoptosis: A review. Journal of biochemical and molecular toxicology, 26, 79-86 (2012).

17. Rokavec, M., Li, H., Jiang, L. \& Hermeking, H. The p53/miR-34 axis in development and disease. Journal of molecular cell biology, 6, 214-230 (2014).

18. Boon, R. A. et al. MicroRNA-34a regulates cardiac ageing and function. Nature, 495, 107-110 (2013).

19. Bernardo, B. C. et al. Therapeutic inhibition of the miR-34 family attenuates pathological cardiac remodeling and improves heart function. Proceedings of the National Academy of Sciences 109, 17615-17620(2012).

20. Yang, Y. et al. MicroRNA-34a Plays a Key Role in Cardiac Repair and Regeneration Following Myocardial InfarctionNovelty and Significance. Circulation research, 117, 450-459 (2015).

21. Piegari, E. et al. MicroRNA-34a regulates doxorubicin-induced cardiotoxicity in rat. Oncotarget, 7, 62312 (2016).

22. Zhu, J. N. et al. Activation of miR-34a-5p/Sirt1/p66shc pathway contributes to doxorubicin-induced cardiotoxicity. Scientific reports, 7, 11879 (2017).

23. Desai, V. G. et al. Early biomarkers of doxorubicin-induced heart injury in a mouse model. Toxicology and applied pharmacology, 281, 221-229 (2014).

24. Piegari, E. et al. Cardioprotective effects of miR-34a silencing in a rat model of doxorubicin toxicity. Sci. Rep, 10, 12250 https://doi.org/10.1038/s41598-020-69038-3 (2020). 
25. Winter, J., Jung, S., Keller, S., Gregory, R. I. \& Diederichs, S. Many roads to maturity: microRNA biogenesis pathways and their regulation. Nature cell biology, 11, 228 (2009).

26. Slabáková, E., Culig, Z., Remšík, J. \& Souček, K. Alternative mechanisms of miR-34a regulation in cancer. Cell death \& disease, 8, e3100-e3100 (2017).

27. Kawai, S. \& Amano, A. BRCA1 regulates microRNA biogenesis via the DROSHA microprocessor complex. Journal of Cell Biology, 197, 201-208 (2012).

28. Wang, X., Zhao, X., Gao, P. \& Wu, M. c-Myc modulates microRNA processing via the transcriptional regulation of Drosha. Scientific reports, 3, 1942 (2013).

29. Yu, Z. et al. Cyclin D1 induction of Dicer governs microRNA processing and expression in breast cancer. Nature communications, 4, 1-10 (2013).

30. Frères, P. et al. Neoadjuvant chemotherapy in breast cancer patients induces miR-34a and miR-122 expression. Journal of cellular physiology, 230, 473-481 (2015).

31. Lakhani, H. V. et al. Detecting early onset of anthracyclines-induced cardiotoxicity using a novel panel of biomarkers in West-Virginian population with breast cancer. Sci. Rep, 11, 7954 https://doi.org/10.1038/s41598-021-87209-8 (2021).

32. Frères, $\mathrm{P}$. et al. Variations of circulating cardiac biomarkers during and after anthracycline-containing chemotherapy in breast cancer patients. BMC cancer, 18, 102 (2018).

33. Yang, S. et al. MicroRNA-34 suppresses breast cancer invasion and metastasis by directly targeting Fra-1., 32, 4294-4303 (2013).

34. He, L. et al. A microRNA component of the p53 tumour suppressor network. Nature, 447, 1130 (2007).

35. Raver-Shapira, N. et al. Transcriptional activation of miR-34a contributes to p53-mediated apoptosis. Molecular cell, 26, 731-743 (2007).

36. Salzman, D. W. et al. miR-34 activity is modulated through 5 '-end phosphorylation in response to DNA damage. Nature communications, 7, 1-9 (2016).

37. Tanindi, A. et al. Assessment of right ventricular functions during cancer chemotherapy. European Journal of Echocardiography, 12, 834-840 (2011).

38. Esfahani, M. A., Mokarian, F. \& Karimipanah, M. Alterations in the echocardiographic variables of the right ventricle in asymptomatic patients with breast cancer during anthracycline chemotherapy. Postgraduate medical journal, 93, 271-274 (2017).

39. Tadic, M., Cuspidi, C., Hering, D., Venneri, L. \& Danylenko, O. The influence of chemotherapy on the right ventricle: did we forget something? Clinical cardiology(2017).

40. Keramida, K. \& Farmakis, D. Right ventricular involvement in cancer therapy-related cardiotoxicity: the emerging role of strain echocardiography. Heart Failure Reviews, 1-5(2020).

41. Planek, M. I. C. et al. Prediction of doxorubicin cardiotoxicity by early detection of subclinical right ventricular dysfunction. Cardio-Oncology, 6, 1-8 (2020).

42. Lang, R. M. et al. Recommendations for cardiac chamber quantification by echocardiography in adults: an update from the American Society of Echocardiography and the European Association of 
Cardiovascular Imaging. European Heart Journal-Cardiovascular Imaging, 16, 233-271 (2015).

43. Livak, K. J. \& Schmittgen, T. D. Analysis of relative gene expression data using real-time quantitative PCR and the $2-\Delta \Delta C T$ method. methods 25, 402-408(2001).

Figures

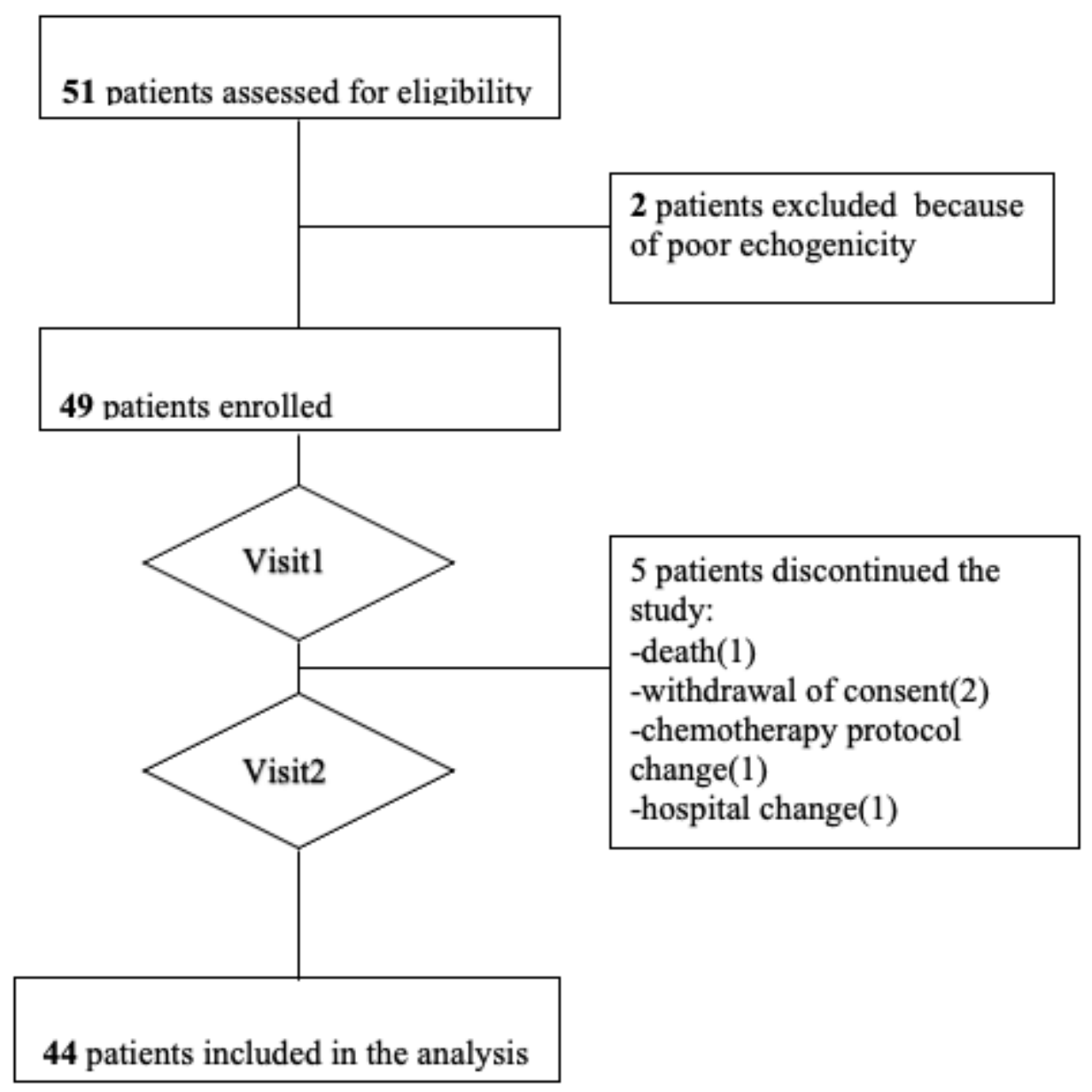

Figure 1

Patient flow chart. 


\section{miR34a and pre-miR34a changes}

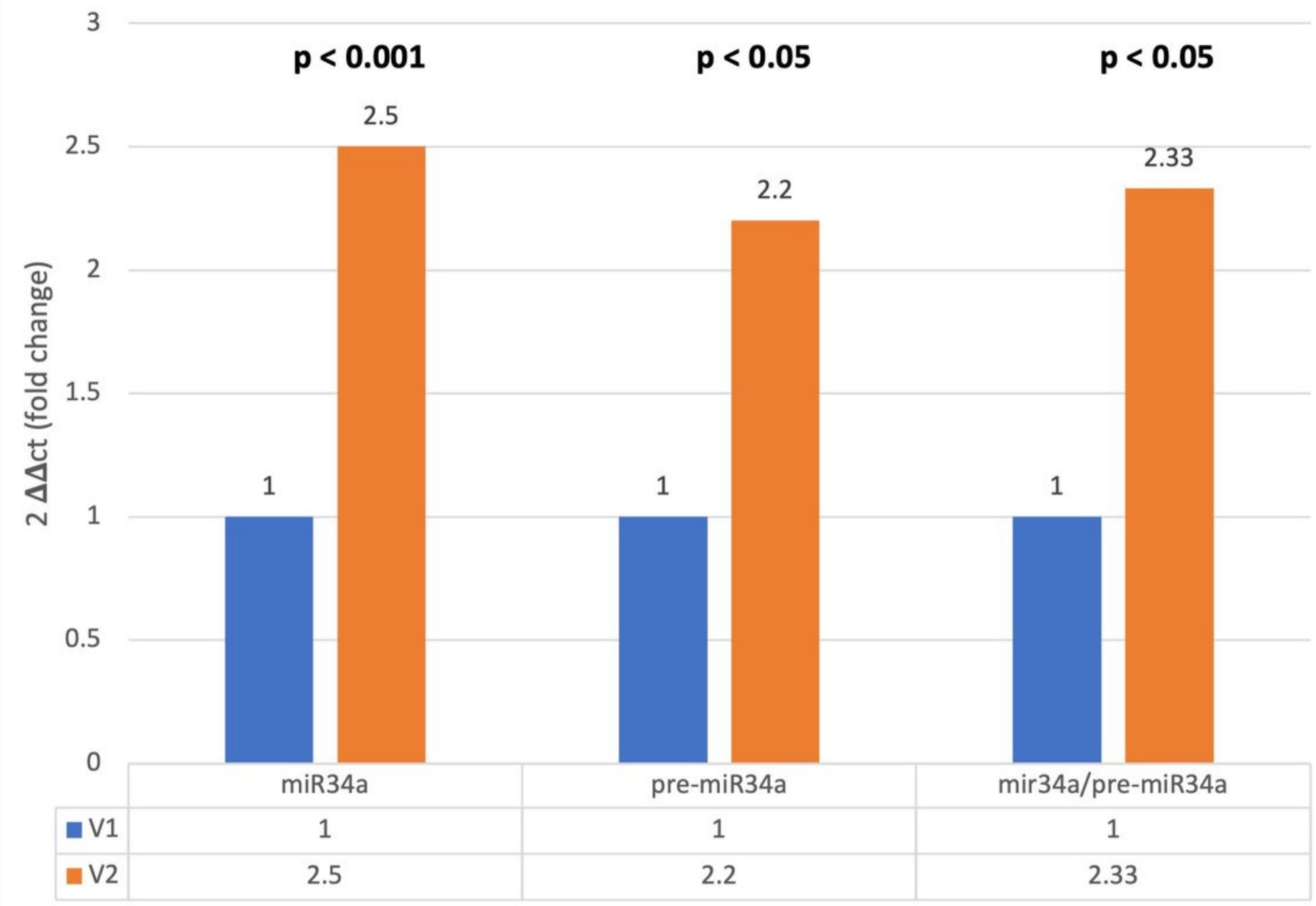

\section{Figure 2}

miR34a and pre-miR34a changes after treatment

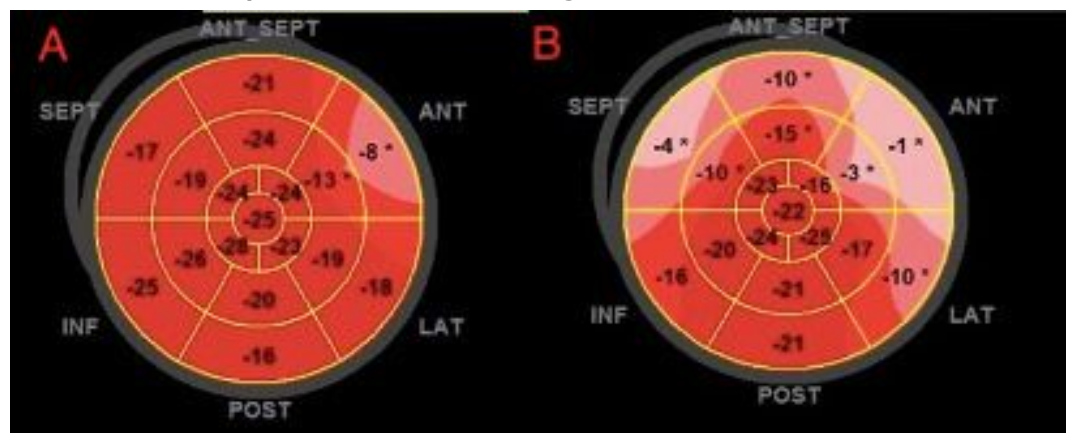

Figure 3

A figure depicting one of patient's LVGLS analysis. A: baseline LVGLS: $20.6 \%$. B: after treatment LVGLS: $15.3 \%$; change in LVGLS: $25 \%$. (LVGLS: left ventricular global longitudinal strain) 


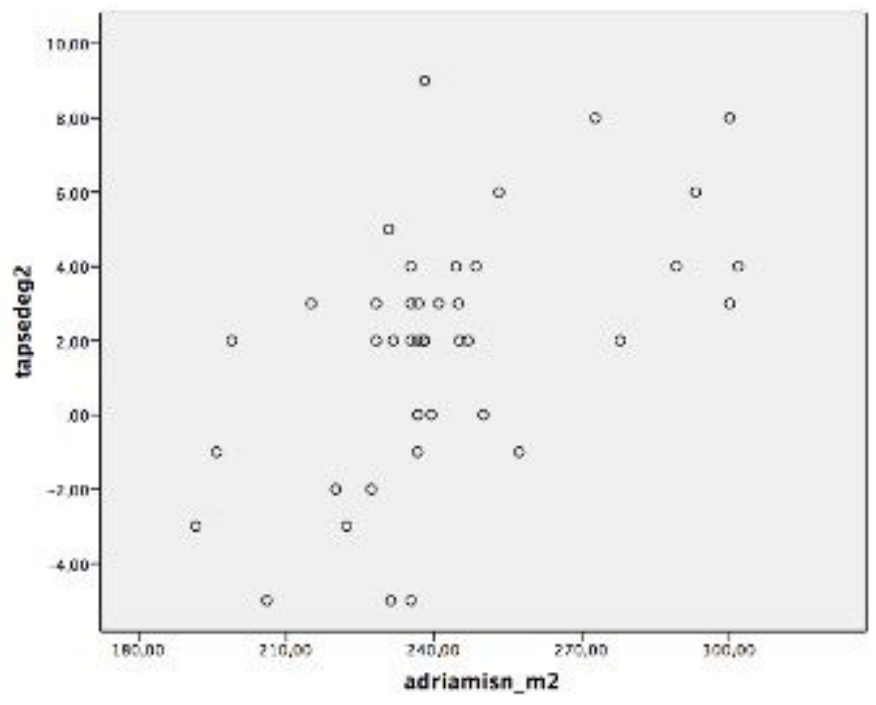

Figure 4

Cumulative Adriamycin Dose (mg/m2) -TAPSE Correlation Curve 